\title{
DEPOSIÇÃO DE CALDA NA APLICAÇÃO AÉREA E TERRESTRE DE FITOSSANITÁRIO NA CULTURA DA BATATA
}

\section{MARIANA R. BUENO ${ }^{1}$, JOÃO PAULO A. R. DA CUNHA ${ }^{2}$, GUILHERME S. ALVES ${ }^{3}$}

RESUMO: O objetivo deste trabalho foi avaliar a deposição de calda pulverizada na cultura da batata e o espectro de gotas promovidos pela aplicação aérea e terrestre. O experimento foi repetido em duas épocas, em lavouras comerciais de batata, no delineamento inteiramente casualizado, em esquema fatorial $6 \times 2$, sendo seis formas de aplicação (aérea, a 15 e $30 \mathrm{~L} \mathrm{ha}^{-1}$ com atomizador rotativo, e terrestre, a 200 e $400 \mathrm{~L} \mathrm{ha}^{-1}$, com pontas de jato plano duplo, com indução de ar e jato cônico vazio); e duas composições de calda (água e água mais o adjuvante fosfatidilcoline + ácido propiônico). Avaliaram-se deposição de calda no dossel da cultura, perdas para o solo e deriva, pela técnica da adição de traçador para quantificação por espectrofotometria, e espectro de gotas, por meio da análise de papéis hidrossensíveis. A adição do adjuvante, de forma geral, proporcionou maior retenção de calda nas folhas inferiores e superiores. A diminuição do volume de aplicação proporcionou boa deposição de calda no alvo, sendo uma alternativa para aumento da capacidade operacional. A aplicação aérea mostrou-se tão eficiente quanto as aplicações terrestres na deposição de calda no alvo. A utilização do adjuvante não interferiu no diâmetro da mediana volumétrica e na percentagem do volume de gotas pulverizadas menores que $100 \mu \mathrm{m}$.

PALAVRAS-CHAVE: aviação agrícola, deriva, ponta de pulverização, espectro de gotas.

\section{SPRAY LIQUID DEPOSITION IN AERIAL AND GROUND PESTICIDE APPLICATION IN POTATO CROP}

\begin{abstract}
The aim of this study was to evaluate the sprayed liquid deposition in potato crop and the droplet spectrum, promoted by aerial and ground applications. The experiment was conducted in duplicate, in commercial potato crops, in completely randomized design, in a factorial model $6 \times 2$ : six application methods (aerial, with rotative atomizer at 15 and $30 \mathrm{~L} \mathrm{ha}^{-1}$, and ground, at 200 and $400 \mathrm{~L} \mathrm{ha}^{-1}$ with air induction twin flat-fan and hollow-cone nozzles) and with two spray liquid compositions (water and water plus phosphatydilcoline + propionic acid adjuvant). It was evaluated spray liquid deposition in the crop canopy, run-off and drift, by addition of a tracer for quantification by spectrophotometry technique and the droplet spectrum by image analysis of watersensitive papers. In general, the adjuvant addition provided a better spray liquid retention into lower and upper leaves. The decrease of spray volume provided a good spray liquid deposition on target that was contribute for an increase of the operational capacity. The aerial application was as efficient as the ground applications on the spray liquid deposition on the target. The use of adjuvant did not modify the volume median diameter and the percentage of spray volume in droplets smaller than $100 \mu \mathrm{m}$ diameter.
\end{abstract}

KEYWORDS: aerial application, drift, spray nozzle, droplet spectrum.

\footnotetext{
${ }^{1}$ Enga. Agrônoma, Doutoranda em Agronomia, Instituto de Ciências Agrárias - ICIAG, Universidade Federal de Uberlândia - UFU, Uberlândia - MG, marianarb_agro@yahoo.com.br.

${ }^{2}$ Eng $^{\circ}$. Agrícola, Prof ${ }^{\circ}$. Doutor, ICIAG - UFU, Uberlândia - MG, jpcunha@iciag.ufu.br.

${ }^{3}$ Eng $^{\circ}$. Agrônomo, Mestrando em Agronomia, ICIAG - UFU, Uberlândia - MG, guilhermeagro43@ yahoo.com.br.

Recebido pelo Conselho Editorial em: 21-3-2012

Aprovado pelo Conselho Editorial em: 26-7-2013
} 


\section{INTRODUÇÃO}

A cultura da batata (Solanum tuberosum L.) é uma das mais exigentes quanto à necessidade de aplicação de produtos fitossanitários. Isto implica estudar métodos mais eficientes de controle de pragas, doenças e plantas infestantes, o que está diretamente relacionado com a tecnologia de aplicação empregada, reduzindo os custos e os riscos ao ambiente. A escolha do método de pulverização é fundamental para que se obtenha uma gota de tamanho ideal, somada ao momento correto de aplicação, compondo um conjunto de fatores que devem ser considerados na tomada de decisão para o controle do alvo (FARINHA et al., 2009).

$\mathrm{Na}$ cultura da batata, é bastante comum a utilização de volumes de calda de $400 \mathrm{~L} \mathrm{ha}^{-1}$ nas aplicações terrestres e $30 \mathrm{~L} \mathrm{ha}^{-1}$ nas aplicações aéreas. Entretanto, são necessários estudos que visem à redução desses volumes, de forma que não se prejudique a eficiência da aplicação.

Bauer et al. (2006) já comentavam a necessidade de redução nos volumes de calda, principalmente com a finalidade de diminuir os custos de produção, reduzir o impacto ambiental e elevar a capacidade operacional dos equipamentos de aplicação. Para atender a essa necessidade, houve grande evolução nos equipamentos de aplicação, inclusive de pontas, com diversas características de pulverização, distribuição e tamanho de gotas.

O processo de formação de gotas pode ser alterado não só pelo tipo de ponta utilizado, mas também pela adição de adjuvantes à calda de aplicação (THEBALDI et al., 2009). O uso correto dos adjuvantes pode aumentar o desempenho do produto aplicado, mas o aumento na eficiência da aplicação também pode aumentar o impacto ambiental pela presença da molécula do adjuvante no ambiente (LAN et al., 2007; RYCKAERT et al., 2007).

Os pulverizadores hidráulicos terrestres são os equipamentos mais comuns para aplicação de fitossanitários. Contudo, apresentam alguns inconvenientes, como perda por amassamento e a compactação do solo, além de terem menor capacidade operacional quando comparados às aeronaves agrícolas. Essas, por sua vez, têm demonstrado grande aplicabilidade, principalmente pela alta capacidade operacional. O grande entrave quanto ao seu uso é a falta de informações quanto à eficiência das aplicações, em comparação às aplicações terrestres (CUNHA et al., 2011).

A deposição de calda de maneira eficiente, segura e econômica nas aplicações hidráulicas só é possível quando se dispõe de pontas que produzem distribuição de calda homogênea e gotas de tamanho e número adequados (NUYTTENS et al., 2007; CUNHA \& SILVA, 2010). O mesmo princípio se aplica às aeronaves agrícolas, seja a barra dotada de pontas, seja de atomizadores rotativos. Esses, por sua vez, produzem gotas mais uniformes em comparação às pontas, permitindo que se trabalhe com menores volumes de calda e, assim, com potencial para redução de perdas para o solo e deriva (GULER et al., 2007).

O objetivo do trabalho foi avaliar a deposição de calda, as perdas para o solo e por deriva, e o espectro de gotas na pulverização da cultura da batata, proporcionadas pela aplicação aérea e terrestre, em diferentes condições operacionais e com duas composições de calda.

\section{MATERIAL E MÉTODOS}

O trabalho foi conduzido na Fazenda Água Santa - Grupo Rocheto, situada no município de Perdizes-MG. As análises laboratoriais foram realizadas no Laboratório de Mecanização Agrícola, do Instituto de Ciências Agrárias da Universidade Federal de Uberlândia, em Uberlândia-MG.

O experimento foi realizado em duplicata de forma semelhante, empregando duas áreas de pivô central (área 1 e área 2). Empregou-se o sistema de plantio convencional, cultivado com batata cultivar Asterix (120 dias de ciclo), em espaçamento de 0,38 m entre plantas, 0,8 m entre linhas e com profundidade de $0,12 \mathrm{~m}$. Na área 1 , no momento das aplicações, a cultura encontrava-se no estádio III, e na área 2, no estádio IV, conforme escala proposta por FILGUEIRA (2008).

Em cada área, o experimento foi conduzido em delineamento inteiramente casualizado, com 
quatro repetições, em esquema fatorial 6 x 2, sendo seis formas de aplicação e duas composições de calda, totalizando 12 tratamentos, conforme descritos na Tabela 1. As aplicações foram realizadas em uma sequência casualizada e a área experimental apresentava homogeneidade.

TABELA 1. Descrição dos tratamentos experimentais. Specifications of experimental treatments.

\begin{tabular}{|c|c|c|c|}
\hline Tratamentos & $\begin{array}{c}\text { Volume de } \\
\text { Calda }\left(\mathrm{L} \mathrm{ha}^{-1}\right)\end{array}$ & & $\begin{array}{c}\text { Pressão de } \\
\text { Trabalho }\end{array}$ \\
\hline Aérea 15 - Atomizador Rotativo & 30 & \multirow{3}{*}{$\begin{array}{l}\text { Com adjuvante } \\
\text { Sem adjuvante } \\
\text { Com adjuvante } \\
\text { Sem adjuvante }\end{array}$} & \multirow{3}{*}{$186 \mathrm{kPa}$} \\
\hline Micronair AU 5000 & 30 & & \\
\hline $\begin{array}{c}\text { Aérea } 30 \text { - Atomizador Rotativo } \\
\text { Micronair AU } 5000\end{array}$ & 15 & & \\
\hline $\begin{array}{c}\text { Terrestre - } 200 \text { C - Ponta de jato cônico vazio } \\
(\mathrm{MAG}-2)\end{array}$ & 200 & \multirow{4}{*}{$\begin{array}{l}\text { Com adjuvante } \\
\text { Sem adjuvante } \\
\text { Com adjuvante } \\
\text { Sem adjuvante } \\
\text { Com adjuvante } \\
\text { Sem adjuvante } \\
\text { Com adjuvante } \\
\text { Sem adjuvante }\end{array}$} & \multirow{2}{*}{$483 \mathrm{kPa}$} \\
\hline $\begin{array}{l}\text { Terrestre - } 400 \text { C - Ponta de jato cônico vazio } \\
\qquad(\mathrm{MAG}-4)\end{array}$ & 400 & & \\
\hline $\begin{array}{c}\text { Terrestre - } 200 \text { IA - Ponta de jato plano duplo com } \\
\text { indução de ar (AD-IA/D } 110 \text { 02) }\end{array}$ & 200 & & \multirow[b]{2}{*}{$207 \mathrm{kPa}$} \\
\hline $\begin{array}{c}\text { Terrestre - } 400 \text { IA - Ponta de jato plano duplo com } \\
\text { indução de ar (AD-IA/D } 110 \text { 04) }\end{array}$ & 400 & & \\
\hline
\end{tabular}

A calda foi composta por água ou água mais o adjuvante fosfatidilcoline + ácido propiônico, cujo nome comercial é LI 700, na concentração recomendada de 0,50\% v/v. De acordo com o fabricante, trata-se de um adjuvante não iônico redutor de tensão superficial e antideriva.

Nas aplicações terrestres, foi utilizado um pulverizador costal de pressão constante $\left(\mathrm{CO}_{2}\right)$, dotado de barra, com quatro bicos espaçados de $0,5 \mathrm{~m}$ entre si. A altura da barra em relação à cultura foi de $0,5 \mathrm{~m}$, e a velocidade de deslocamento foi de $4 \mathrm{~km} \mathrm{~h}^{-1}$. As parcelas experimentais constaram de $51,2 \mathrm{~m}^{2}$, sendo $6,4 \mathrm{~m}$ de largura e $8 \mathrm{~m}$ de comprimento, e foi mantida sempre uma distância longitudinal de $6 \mathrm{~m}$ entre as parcelas. A área útil da parcela foi de 19,2 $\mathrm{m}^{2}$; para isso, descartaram-se duas linhas de cada lado da parcela e um metro de cada extremidade.

Nas aplicações aéreas, foi utilizada uma aeronave agrícola Cessna AG TRUCK - 300, dotada de oito atomizadores rotativos de tela Micronair AU 5000. A altura de voo foi de $3 \mathrm{~m}$ em relação à cultura, a velocidade de aplicação, de $177 \mathrm{~km} \mathrm{~h}^{-1}$, e o ângulo das pás do atomizador, de $45^{\circ}$. $\mathrm{O}$ tamanho das parcelas foi de $19.200 \mathrm{~m}^{2}$, correspondente a $300 \mathrm{~m}$ de comprimento e $64 \mathrm{~m}$ de largura, equivalente a quatro passadas de $16 \mathrm{~m}$ do avião. Após a aplicação, foi estabelecida uma distância lateral de $50 \mathrm{~m}$ entre cada parcela. A área útil correspondeu a $1.920 \mathrm{~m}^{2}$; para isso, descartaram-se 20 $\mathrm{m}$ de cada lado e $110 \mathrm{~m}$ de cada extremidade das parcelas.

As aplicações terrestres e aéreas foram realizadas sempre na direção perpendicular ao vento. $\mathrm{Na}$ primeira aplicação, as condições ambientais permaneceram dentro dos seguintes intervalos: umidade relativa do ar entre 58,5 e $64 \%$, temperatura do ar entre 21 e $24^{\circ} \mathrm{C}$ e velocidade do vento entre 4,3 e 8,6 $\mathrm{km} \mathrm{h}^{-1}$. Na segunda aplicação, as condições foram: umidade relativa do ar entre 60,5 e $68,7 \%$, temperatura do ar entre 24 e $26,5^{\circ} \mathrm{C}$ e velocidade do vento entre 5 e $15,5 \mathrm{~km} \mathrm{~h}^{-1}$. Esses dados foram obtidos por meio de um termo-higroanemômetro digital (Kestrel ${ }^{\circledR} 4000$ ).

Avaliaram-se a deposição de calda de pulverização na metade inferior e superior da cultura da batata, as perdas para o solo e a deriva. Calculou-se a diferença percentual entre a deposição de calda nas folhas superiores e inferiores. E avaliou-se também o espectro das gotas pulverizadas por meio da análise de papéis hidrossensíveis. 
Para a avaliação do espectro de gotas, antes da pulverização, foram colocados aleatoriamente em cada parcela quatro papéis hidrossensíveis $(76 \times 26 \mathrm{~mm})$, na posição horizontal, suspensos em uma haste metálica imediatamente acima das plantas. Em laboratório, os papéis foram digitalizados com resolução de 600 dpi não interpolados, com cores em 24 bits, e analisados por meio do programa computacional CIR® 1.5 - Conteo y Tipificación de Impactos de Pulverización.

Foram estudados os parâmetros: $\mathrm{Dv}_{0,5}$ - diâmetro de gota, tal que $50 \%$ do volume do líquido pulverizado é constituído de gotas de tamanho menor que esse valor, também conhecido como diâmetro da mediana volumétrica (DMV), e $\mathrm{Dv}<100 \mu \mathrm{m}$ - percentagem do volume aplicado cujas gotas possuam diâmetro inferior a $100 \mu \mathrm{m}$.

Para a avaliação dos depósitos, utilizou-se de um traçador composto do corante alimentício azul, catalogado internacionalmente pela "Food, Drug \& Cosmetic", como FD\&C Blue n.1 (azul brilhante), na dose de $0,4 \mathrm{~kg} \mathrm{ha}^{-1}$ para ser detectado por absorbância em espectrofotometria. Para tanto, foi utilizado um espectrofotômetro (fotômetro fotoelétrico de filtro), com lâmpada de tungstênio-halogênio. A quantificação da coloração foi feita por absorbância em $630 \mathrm{~nm}$, faixa de detecção do corante utilizado, conforme metodologia apresentada por PALLADINI et al. (2005). De acordo com PINTO et al. (2007), o traçador Azul Brilhante é estável por um período de 5 horas de exposição solar.

Após a pulverização, foram selecionadas dez plantas escolhidas ao acaso, na área útil de cada parcela, e em cada planta, duas folhas coletadas: uma no terço superior e outra no terço inferior. As folhas foram agrupadas por posição na planta e colocadas em recipientes plásticos contendo $0,1 \mathrm{~L}$ de água destilada. Esses recipientes foram fechados, agitados por 30 segundos e acondicionados imediatamente em caixa térmica, sob ausência de luz, para posterior leitura da absorbância em laboratório. A área das folhas foi medida pelo programa de análise de imagens "Image Tool" (University of Texas, Texas, USA), após serem digitalizadas com resolução espacial de 600 dpi não interpolados, com cores em 24 bits. Com o uso das curvas de calibração, obtidas por meio de soluções-padrão, os dados de absorbância foram transformados para a unidade de massa do traçador em relação à área foliar $\left(\mu \mathrm{g} \mathrm{cm}^{-2}\right)$.

Para determinar as perdas de calda no solo, foram colocadas em cada repetição quatro lâminas de vidro (com área de $37,24 \mathrm{~cm}^{2}$ cada) próximas ao caule da planta, em contato com a superfície do solo. Após a aplicação, as lâminas foram recolhidas e colocadas em recipientes de plástico contendo $0,1 \mathrm{~L}$ de água destilada. A quantificação do traçador foi feita de forma semelhante à realizada nas folhas.

Com relação à análise de deriva, foram colocados quatro fios de polietileno de $4 \mathrm{~m}$ de comprimento e $2 \mathrm{~mm}$ de diâmetro, a uma altura de $2 \mathrm{~m}$ em relação ao solo, a uma distância de $5 \mathrm{~m}$, externamente paralelos à borda da parcela, no sentido de deslocamento do vento. Após a aplicação, os fios foram colocados em sacos plásticos com 0,1 L de água destilada, para posterior quantificação da coloração por espectrofotometria.

A perda por deriva nas aplicações aéreas não foi calculada em vista da dificuldade de se determinar, com exatidão, o limite final das parcelas para disposição dos marcos coletores. No entanto, estimou-se o percentual de perdas por deriva de todos os tratamentos, de forma indireta, pela utilização de papéis hidrossensíveis, calculando-se a percentagem de gotas menores que 100 $\mu \mathrm{m}$. De acordo com MILLER \& BUTLER-ELLIS (2000), esta é uma medida simples que permite determinar o risco de deriva.

Os dados foram primeiramente submetidos aos testes de normalidade de Shapiro Wilk e homogeneidade das variâncias de Levene, utilizando-se do programa SPSS 16. Em seguida, procedeu-se à análise de variância e, constatada diferença significativa, através do programa estatístico SISVAR, as médias foram comparadas pelo teste Tukey, a 0,05 de probabilidade. 


\section{RESULTADOS E DISCUSSÃO}

\section{Primeira Aplicação - Área 1}

Ao se avaliar o efeito das formas e dos volumes de aplicação na deposição de calda nas folhas inferiores, nota-se que não houve diferença entre os tratamentos (Tabela 2). Já para as folhas superiores das plantas, as aplicações aéreas com 15 e $30 \mathrm{~L} \mathrm{ha}^{-1}$ proporcionaram retenção de líquido semelhante às aplicações terrestres com menor volume de calda $\left(200 \mathrm{~L} \mathrm{ha}^{-1}\right)$, independentemente do tipo de ponta.

TABELA 2. Deposição de calda nas folhas inferiores e superiores das plantas de batata, após a primeira aplicação aérea e terrestre, com e sem adjuvante adicionado à calda. Spray liquid deposition into lower and upper leaves of potato plants after the first aerial and ground application, with and without adjuvant addition in the spray liquid.

\begin{tabular}{|c|c|c|c|c|c|c|}
\hline \multirow{3}{*}{$\begin{array}{l}\text { Formas e volumes } \\
\text { de aplicação }\left(\mathrm{L} \mathrm{ha}^{-1}\right)\end{array}$} & \multicolumn{3}{|c|}{ Folhas inferiores $\left(\mu \mathrm{g} \mathrm{cm}^{-2}\right)$} & \multicolumn{3}{|c|}{ Folhas superiores $\left(\mu \mathrm{g} \mathrm{cm}^{-2}\right)$} \\
\hline & \multicolumn{2}{|c|}{ Adjuvante } & \multirow{2}{*}{ Média } & \multicolumn{2}{|c|}{ Adjuvante } & \multirow{2}{*}{ Média } \\
\hline & Sem & Com & & Sem & Com & \\
\hline Aérea - 15 & 0,207 & 0,308 & $0,257 \mathrm{~A}$ & 0,570 & 0,680 & $0,625 \mathrm{AB}$ \\
\hline Aérea - 30 & 0,308 & 0,252 & $0,280 \mathrm{~A}$ & 0,679 & 0,760 & $0,719 \mathrm{~A}$ \\
\hline Terrestre - $200 \mathrm{C}^{\prime}$ & 0,316 & 0,384 & $0,350 \mathrm{~A}$ & 0,691 & 0,633 & $0,662 \mathrm{AB}$ \\
\hline Terrestre - $400 \mathrm{C}$ & 0,211 & 0,353 & $0,282 \mathrm{~A}$ & 0,488 & 0,599 & $0,543 \mathrm{BC}$ \\
\hline Terrestre - 200 IA" & 0,311 & 0,339 & $0,325 \mathrm{~A}$ & 0,595 & 0,684 & $0,639 \mathrm{AB}$ \\
\hline Terrestre - 400 IA & 0,276 & 0,311 & $0,293 \mathrm{~A}$ & 0,391 & 0,518 & $0,455 \mathrm{C}$ \\
\hline \multirow[t]{3}{*}{ Média } & $0,271 \mathrm{~b}$ & $0,325 \mathrm{a}$ & & $0,569 \mathrm{~b}$ & $0,646 \mathrm{a}$ & \\
\hline & \multicolumn{3}{|c|}{$\mathrm{CV}=29,16 \%$} & \multicolumn{3}{|c|}{$\mathrm{CV}=17,93 \%$} \\
\hline & $\mathrm{F}_{\mathrm{F}}=1,211^{\mathrm{ns}}$ & $\mathrm{F}_{\mathrm{A}}=4,492 *$ & $\mathrm{~F}_{\mathrm{FxA}}=1,230^{\mathrm{ns}}$ & $\mathrm{F}_{\mathrm{F}}=5,969 * *$ & $\mathrm{~F}_{\mathrm{A}}=5,947 *$ & $\mathrm{~F}_{\mathrm{FxA}}=0,780^{\mathrm{ns}}$ \\
\hline $\begin{array}{l}\text { 'C: ponta de jato cônico } \\
\text { calculado para o fator for } \\
\text { a interação entre os fator } \\
\text { e minúsculas, nas linhas, } \\
\text { ns não significativo. }\end{array}$ & -IA- nont. & to & $\begin{array}{l}\text { com induçã } \\
\text { o F calculado } \\
\text { ıvante. Médic }\end{array}$ & $\begin{array}{l}\text { ar; CV: coefic } \\
\text { a o fator adjuva }\end{array}$ & $\begin{array}{l}\text { ciente de variaçe } \\
\text { nte; } \mathrm{F}_{\text {FxA }}: \text { valor } \\
\text { s distintas maiús }\end{array}$ & $\begin{array}{l}\text { o; } \mathrm{F}_{\mathrm{F}} \text { : valor do } \mathrm{F} \\
\text { o F calculado para } \\
\text { culas, nas colunas, }\end{array}$ \\
\hline
\end{tabular}

BAYER et al. (2011), em contrapartida, estudando diferentes volumes de aplicação aérea na cultura do arroz, encontraram diferenças quanto à deposição no terço inferior das plantas, com maior retenção de líquido para os menores volumes de calda. Possivelmente, a diferença de arquitetura entre as duas culturas seja a justificativa para tal diferença.

As aplicações com maior volume de calda $\left(400 \mathrm{~L} \mathrm{ha}^{-1}\right)$ resultaram em menor retenção de calda na folhagem superior, diferindo do trabalho de OZEKI (2006). O autor afirmou que, nas aplicações com volumes maiores, as gotas resultantes da pulverização tendem a se estabelecer na parte superior da planta, diminuindo a retenção na parte mais interna do dossel. Contudo, espera-se que maiores volumes causem maior escorrimento, o que pode explicar o resultado obtido.

A adição do adjuvante fosfatidilcoline + ácido propiônico proporcionou maior retenção de calda, tanto nas folhas inferiores quanto nas folhas superiores, o que confirma que esses produtos podem melhorar a aderência e a penetração da calda de pulverização no interior das culturas (MENDONÇA et al. 2007; RYCKAERT et al., 2007). Utilizando o mesmo adjuvante, VAN ZYL et al. (2010) obtiveram resultado similar em videira.

Ao analisar a uniformidade de distribuição de líquido, verifica-se, na Tabela 3, que a menor diferença entre a quantidade de calda retida nas folhas superiores e inferiores foi encontrada quando se aplicaram $400 \mathrm{~L} \mathrm{ha}^{-1}$ com a ponta AD-IA/D 110 04. No entanto, esta não diferiu dos demais tratamentos de aplicação terrestre. 
TABELA 3. Diferença entre a deposição de calda nas folhas superiores e inferiores das plantas de batata, após a primeira aplicação aérea e terrestre, com e sem adjuvante adicionado à calda. Difference between the spray liquid deposition into lower and upper leaves of potato plants, after the first aerial and ground application, with and without adjuvant addition in the spray liquid.

\begin{tabular}{|c|c|c|c|}
\hline \multirow{3}{*}{ Formas e volumes de aplicação $\left(\mathrm{L} \mathrm{ha}^{-1}\right)$} & \multicolumn{3}{|c|}{ Diferença $(\%)$} \\
\hline & \multicolumn{2}{|c|}{ Adjuvante } & \multirow{2}{*}{ Média } \\
\hline & Sem & Com & \\
\hline Aérea - 15 & 62,86 & 54,41 & $58,64 \mathrm{~B}$ \\
\hline Aérea - 30 & 54,41 & 67,07 & $60,74 \mathrm{~B}$ \\
\hline Terrestre - $200 \mathrm{C}^{\prime}$ & 53,51 & 38,86 & $46,18 \mathrm{AB}$ \\
\hline Terrestre - $400 \mathrm{C}$ & 51,57 & 42,50 & $47,03 \mathrm{AB}$ \\
\hline Terrestre - 200 IA" & 48,13 & 49,00 & $48,56 \mathrm{AB}$ \\
\hline Terrestre - 400 IA & 29,53 & 38,14 & $33,84 \mathrm{~A}$ \\
\hline \multirow[t]{2}{*}{ Média } & $50,00 \mathrm{a}$ & $48,33 \mathrm{a}$ & \\
\hline & $\mathrm{F}_{\mathrm{F}}=3,225^{*}$ & $\begin{array}{l}\mathrm{F}=31,14 \% \\
\mathrm{~F}_{\mathrm{A}}=0,143^{\mathrm{ns}}\end{array}$ & $\mathrm{F}_{\mathrm{FxA}}=1,001^{\mathrm{ns}}$ \\
\hline
\end{tabular}

'C: ponta de jato cônico vazio; "IA: ponta de jato plano duplo com indução de ar; $C V$ : coeficiente de variação; $\mathrm{F}_{\mathrm{F}}$ : valor do $\mathrm{F}$ calculado para o fator formas e volumes de aplicação; $\mathrm{F}_{\mathrm{A}}$ : valor do $\mathrm{F}$ calculado para o fator adjuvante; $\mathrm{F}_{\mathrm{FxA}}$ : valor do $\mathrm{F}$ calculado para a interação entre os fatores formas e volumes de aplicação e adjuvante. Médias seguidas por letras distintas maiúsculas, nas colunas, e minúsculas, nas linhas, diferem entre si, pelo teste de Tukey, a 0,05 de probabilidade. $* *$ significativo a 0,$01 ; *$ significativo a 0,05 ;

${ }^{\text {ns }}$ não significativo.

Os dois tratamentos de aplicação aérea proporcionaram a maior desuniformidade de deposição de gotas entre o dossel superior e inferior das plantas, mas sem se diferenciarem dos tratamentos terrestres, com exceção da aplicação com $400 \mathrm{~L} \mathrm{ha}^{-1}$ e ponta de indução de ar. Com relação à adição de adjuvante, a ausência ou a presença desse na calda não interferiu na diferença entre as deposições nas folhas inferiores e superiores das plantas de batata.

De forma geral, a deposição nas folhas inferiores da cultura foi menor que nas folhas superiores. No momento da aplicação, as plantas de batata encontravam-se no máximo desenvolvimento vegetativo. O índice de área foliar (IAF) da planta é parte importante na qualidade da aplicação. Plantas bem desenvolvidas possuem maior área foliar a ser protegida e, consequentemente, impõem maior dificuldade à deposição das gotas na pulverização (BOLLER et al., 2007), principalmente na parte inferior das plantas.

Para a deriva, avaliada nos fios de polietileno, os tratamentos terrestres com volumes de $200 \mathrm{e}$ $400 \mathrm{~L} \mathrm{ha}^{-1}$ com pontas AD-IA/D 11002 e 110 04, sem adjuvante, resultaram em menores valores (Tabela 4). CUNHA et al. (2005), avaliando a deriva de pontas de jato plano standard e de jato cônico vazio, também verificaram que a pulverização com as pontas de jato cônico proporcionou maior deposição de gotas fora da área-alvo, da mesma forma que o observado neste trabalho.

As pontas de jato cônico vazio, normalmente, produzem grande volume de gotas com diâmetro inferior a $100 \mu \mathrm{m}$, que são mais sujeitas à deriva. Além disso, a turbulência gerada por essas pontas, juntamente com a massa de folhas, pode contribuir com a suspensão das gotas, que ficam mais sujeitas à deriva. Para os tratamentos com pontas de jato cônico, houve redução de deriva quando se adicionou adjuvante à calda, o que não ocorreu para as pontas de jato plano duplo com indução de ar. $\mathrm{O}$ adjuvante conseguiu reduzir as gotas muito finas das pontas de jato cônico vazio, que existem em baixa quantidade nas pontas de indução de ar.

Os tratamentos terrestres com 200 e $400 \mathrm{~L} \mathrm{ha}^{-1}$ com pontas de jato plano duplo, com indução de ar, proporcionaram tendência de maiores valores de perda de calda por escorrimento (avaliada por meio das lâminas no solo), que foi reduzida quando se adicionou adjuvante à calda (Tabela 4). Esse também reduziu o escorrimento para os demais tratamentos, exceto para a aplicação aérea com $15 \mathrm{~L} \mathrm{ha}^{-1}$ de calda. 
TABELA 4. Deriva e perdas para o solo, após a primeira aplicação aérea e terrestre, com e sem adjuvante adicionado à calda. Drift and spray liquid run-off after the first aerial and ground application, with and without adjuvant addition in the spray liquid.

\begin{tabular}{|c|c|c|c|c|c|c|}
\hline \multirow{3}{*}{$\begin{array}{l}\text { Formas e volumes } \\
\text { de aplicação ( } \mathrm{L} \text { ha } \\
1 \text { ) }\end{array}$} & \multicolumn{3}{|c|}{ Deriva $\left(\mu \mathrm{g} \mathrm{cm}^{-2}\right)$} & \multicolumn{3}{|c|}{ Perdas para o solo $\left(\mu \mathrm{g} \mathrm{cm}^{-2}\right)$} \\
\hline & \multicolumn{2}{|c|}{ Adjuvante } & \multirow[b]{2}{*}{ Média } & \multicolumn{2}{|c|}{ Adjuvante } & \multirow{2}{*}{ Média } \\
\hline & Sem & Com & & Sem & Com & \\
\hline Aérea - 15 & - & - & - & $0,103 \mathrm{Aa}$ & $0,109 \mathrm{ABa}$ & 0,106 \\
\hline Aérea - 30 & - & - & - & $0,269 \mathrm{Bb}$ & $0,122 \mathrm{ABa}$ & 0,196 \\
\hline Terrestre - $200 \mathrm{C}^{\prime}$ & $0,073 \mathrm{Bb}$ & $0,029 \mathrm{Aa}$ & 0,051 & $0,244 \mathrm{ABb}$ & $0,018 \mathrm{Aa}$ & 0,131 \\
\hline Terrestre - $400 \mathrm{C}$ & $0,077 \mathrm{Bb}$ & $0,028 \mathrm{Aa}$ & 0,052 & $0,296 \mathrm{Bb}$ & $0,106 \mathrm{ABa}$ & 0,201 \\
\hline Terrestre - 200 IA" & $0,007 \mathrm{Aa}$ & $0,005 \mathrm{Aa}$ & 0,006 & $0,480 \mathrm{Cb}$ & $0,206 \mathrm{Ba}$ & 0,343 \\
\hline Terrestre - 400 IA & 0,008 Aa & $0,013 \mathrm{Aa}$ & 0,011 & $0,958 \mathrm{Db}$ & $0,250 \mathrm{Ba}$ & 0,604 \\
\hline \multirow[t]{2}{*}{ Média } & 0,041 & 0,019 & & 0,392 & 0,135 & \\
\hline & \multicolumn{3}{|c|}{$\begin{array}{c}\mathrm{CV}=51,37 \% \\
\mathrm{~F}_{\mathrm{F}}=20,947 * * \mathrm{~F}_{\mathrm{A}}=16,968 * * \\
\mathrm{~F}_{\mathrm{FxA}}=6,542 * *\end{array}$} & \multicolumn{3}{|c|}{$\begin{array}{c}\mathrm{CV}=26,00 \% \\
\mathrm{~F}_{\mathrm{F}}=59,012 * * \mathrm{~F}_{\mathrm{A}}=168,065^{* *} \\
\mathrm{~F}_{\mathrm{FxA}}=24,744 * *\end{array}$} \\
\hline
\end{tabular}

'C: ponta de jato cônico vazio; "IA: ponta de jato plano duplo com indução de ar; CV: coeficiente de variação; $\mathrm{F}_{\mathrm{F}}$ : valor do $\mathrm{F}$ calculado para o fator formas e volumes de aplicação; $\mathrm{F}_{\mathrm{A}}$ : valor do $\mathrm{F}$ calculado para o fator adjuvante; $\mathrm{F}_{\mathrm{FxA}}$ : valor do $\mathrm{F}$ calculado para a interação entre os fatores formas e volumes de aplicação e adjuvante. Médias seguidas por letras distintas maiúsculas, nas colunas, e minúsculas, nas linhas, diferem entre si, pelo teste de Tukey, a 0,05 de probabilidade. $* *$ significativo a 0,$01 ; *$ significativo a 0,05 ;

${ }^{\text {ns }}$ não significativo. A deriva para os tratamentos aéreos não foi calculada conforme explicado no item material e métodos.

Os tratamentos de aplicação aérea com 15 e $30 \mathrm{~L} \mathrm{ha}^{-1}$, empregando atomizador rotativo de tela, produziram os menores tamanhos de gota, enquanto os maiores valores foram produzidos pelos tratamentos de aplicação terrestre com 200 e $400 \mathrm{~L} \mathrm{ha}^{-1}$, empregando pontas de jato plano duplo com indução de ar (Tabela 5).

A adição do adjuvante fosfatidilcoline + ácido propiônico à calda não alterou o diâmetro volumétrico das gotas e a percentagem de gotas menores que $100 \mu \mathrm{m}$ (Tabela 5). FERREIRA et al. (2009) também não encontraram diferença no $\mathrm{Dv}_{0,5}$ das gotas produzidas pelas pontas TF-VS-2 (jato plano de grande ângulo) e AI 11003 (jato plano com indução de ar) com a adição de diferentes adjuvantes à calda. No entanto, para a ponta TTI 11003 (jato plano defletor com indução de ar), com a adição dos adjuvantes LI-700 (fosfatidilcoline + ácido propriônico), Aquafol (fertilizante foliar) e Agridex (óleo mineral), os autores obtiveram valores menores de $\mathrm{Dv}_{0,5}$. Isso demonstra que o efeito do adjuvante é dependente do tipo de ponta empregada.

Os tratamentos terrestres com pontas AD-IA/D proporcionaram a menor percentagem de gotas pulverizadas menores que $100 \mu \mathrm{m}$, e os tratamentos aéreos, os maiores valores (Tabela 5). Segundo CUNHA et al. (2003), a interpretação dos dados de percentagem de gotas com diâmetro inferior a $100 \mu \mathrm{m}$ permite a estimativa do potencial de deriva da aplicação. Não existe valor-padrão indicativo de risco de deriva ou de aplicação segura; entretanto, para os autores, valores abaixo de $15 \%$ de volume de gotas com diâmetro inferior a $100 \mu \mathrm{m}$ são mais adequados para aplicações seguras ambientalmente, com menor risco de deriva.

Assim, aplicações aéreas com o espectro de gotas encontrado (101 à $120 \mu \mathrm{m})$ devem ser realizadas, preferencialmente, em condições ambientais favoráveis à aplicação de fitossanitário, a fim de reduzir ao máximo as perdas por deriva. 
TABELA 5. Diâmetro da mediana volumétrica e percentagem do volume pulverizado, composto por gotas com diâmetro inferior a $100 \mu \mathrm{m}$, após a primeira aplicação aérea e terrestre, com e sem adição de adjuvante à calda. Volume median diameter and percentage of spray volume in droplets smaller than $100 \mu \mathrm{m}$ diameter after the first aerial and ground application, with and without adjuvant addition in the spray liquid.

\begin{tabular}{|c|c|c|c|c|c|c|}
\hline \multirow{3}{*}{$\begin{array}{l}\text { Formas e volumes de } \\
\text { aplicação }\left(\mathrm{L} \mathrm{ha}^{-1}\right)\end{array}$} & \multicolumn{3}{|c|}{$\operatorname{Dv}_{0,5}(\mu \mathrm{m})$} & \multicolumn{3}{|c|}{ Gotas $<100 \mu \mathrm{m}(\%)$} \\
\hline & \multicolumn{2}{|c|}{ Adjuvante } & \multirow{2}{*}{ Média } & \multicolumn{2}{|c|}{ Adjuvante } & \multirow{2}{*}{ Média } \\
\hline & Sem & Com & & Sem & Com & \\
\hline Aérea - 15 & 101 & 101 & $101 \mathrm{~A}$ & 49,03 & 47,95 & $48,49 \mathrm{D}$ \\
\hline Aérea - 30 & 132 & 110 & $121 \mathrm{~A}$ & 24,19 & 35,73 & $29,96 \mathrm{C}$ \\
\hline Terrestre - $200 \mathrm{C}^{\prime}$ & 155 & 156 & $155 \mathrm{AB}$ & 11,49 & 12,22 & $11,86 \mathrm{~B}$ \\
\hline Terrestre - $400 \mathrm{C}$ & 179 & 180 & $179 \mathrm{~B}$ & 12,80 & 12,07 & $12,44 \mathrm{~B}$ \\
\hline Terrestre - 200 IA" & 436 & 440 & $438 \mathrm{C}$ & 1,63 & 0,73 & $1,18 \mathrm{~A}$ \\
\hline Terrestre - 400 IA & 480 & 487 & $483 \mathrm{C}$ & 1,72 & 1,56 & $1,64 \mathrm{~A}$ \\
\hline \multirow{4}{*}{ Média } & $247 \mathrm{a}$ & $246 a$ & & $16,81 \mathrm{a}$ & $18,38 \mathrm{a}$ & \\
\hline & \multicolumn{3}{|c|}{$\mathrm{CV}=15,34 \%$} & \multicolumn{3}{|c|}{$\mathrm{CV}=30,29 \%$} \\
\hline & \multirow{2}{*}{\multicolumn{3}{|c|}{$\mathrm{F}_{\mathrm{F}}=159,38^{* *} \quad \mathrm{~F}_{\mathrm{A}}$}} & $\mathrm{F}_{\mathrm{F}}=95$ & $\mathrm{~F}_{\mathrm{A}}=$ & $t^{\mathrm{ns}}$ \\
\hline & & & & \multicolumn{3}{|c|}{$\mathrm{F}_{\mathrm{FxA}}=1,71^{\mathrm{ns}}$} \\
\hline \multicolumn{7}{|c|}{$\begin{array}{l}\text { 'C: ponta de jato cônico vazio; "IA: ponta de jato plano duplo com indução de ar; } \mathrm{CV} \text { : coeficiente de variação; } \mathrm{F}_{\mathrm{F}} \text { : valor do } \mathrm{F} \\
\text { calculado para o fator formas e volumes de aplicação; } \mathrm{F}_{\mathrm{A}} \text { : valor do } \mathrm{F} \text { calculado para o fator adjuvante; } \mathrm{F}_{\mathrm{FxA}} \text { : valor do } \mathrm{F} \text { calculado para } \\
\text { a interação entre os fatores formas e volumes de aplicação e adjuvante. Médias seguidas por letras distintas maiúsculas, nas colunas, } \\
\text { e minúsculas, nas linhas, diferem entre si, pelo teste de Tukey, a } 0,05 \text { de probabilidade. ** significativo a } 0,01 ; * \text { significativo a } 0,05 \text {; }\end{array}$} \\
\hline
\end{tabular}

\section{Segunda Aplicação - Área 2}

Não houve diferença significativa entre os tratamentos de aplicação aérea e terrestre quanto à deposição nas partes inferior e superior da cultura (Tabela 6). Entretanto, quando se utilizou adjuvante na calda, esse proporcionou maior retenção de líquido nas duas posições estudadas.

TABELA 6. Deposição de calda nas folhas inferiores e superiores das plantas de batata, após a segunda aplicação aérea e terrestre, com e sem adjuvante adicionado à calda. Spray liquid deposition into lower and upper leaves of potato plant after the first aerial and ground application, with and without adjuvant addition in the spray liquid.

\begin{tabular}{|c|c|c|c|c|c|c|}
\hline \multirow{3}{*}{$\begin{array}{l}\text { Formas e volumes } \\
\text { de aplicação (L ha } \\
1 \text { ) }\end{array}$} & \multicolumn{3}{|c|}{ Folhas inferiores $\left(\mu \mathrm{g} \mathrm{cm}^{-2}\right)$} & \multicolumn{3}{|c|}{ Folhas superiores $\left(\mu \mathrm{g} \mathrm{cm}^{-2}\right)$} \\
\hline & \multicolumn{2}{|c|}{ Adjuvante } & \multirow{2}{*}{ Média } & \multicolumn{2}{|c|}{ Adjuvante } & \multirow{2}{*}{ Média } \\
\hline & Sem & Com & & Sem & Com & \\
\hline Aérea - 15 & 0,314 & 0,326 & $0,320 \mathrm{~A}$ & 0,401 & 0,488 & $0,445 \mathrm{~A}$ \\
\hline Aérea - 30 & 0,305 & 0,371 & $0,338 \mathrm{~A}$ & 0,558 & 0,618 & $0,588 \mathrm{~A}$ \\
\hline Terrestre - $200 \mathrm{C}^{\prime}$ & 0,250 & 0,485 & $0,368 \mathrm{~A}$ & 0,569 & 0,700 & $0,634 \mathrm{~A}$ \\
\hline Terrestre - $400 \mathrm{C}$ & 0,273 & 0,322 & $0,298 \mathrm{~A}$ & 0,545 & 0,536 & $0,541 \mathrm{~A}$ \\
\hline Terrestre - 200 IA" & 0,423 & 0,445 & $0,434 \mathrm{~A}$ & 0,562 & 0,655 & $0,609 \mathrm{~A}$ \\
\hline Terrestre - 400 IA & 0,266 & 0,295 & $0,281 \mathrm{~A}$ & 0,477 & 0,602 & $0,539 \mathrm{~A}$ \\
\hline \multirow[t]{3}{*}{ Média } & $0,305 \mathrm{~b}$ & $0,374 \mathrm{a}$ & & $0,519 \mathrm{~b}$ & $0,600 \mathrm{a}$ & \\
\hline & \multicolumn{3}{|c|}{$\mathrm{CV}=31,45 \%$} & \multicolumn{3}{|c|}{$\mathrm{CV}=22,97 \%$} \\
\hline & $\mathrm{F}_{\mathrm{F}}=2,152^{\mathrm{ns}}$ & $\mathrm{F}_{\mathrm{A}}=4,972 *$ & $\mathrm{~F}_{\mathrm{FxA}}=1,225^{\mathrm{ns}}$ & $\mathrm{F}_{\mathrm{F}}=2,208^{\mathrm{ns}}$ & $\mathrm{F}_{\mathrm{A}}=4,826^{*}$ & $\mathrm{~F}_{\mathrm{FxA}}=0,319^{\mathrm{ns}}$ \\
\hline
\end{tabular}


SCUDELER et al. (2004), estudando a influência de pontas de pulverização hidráulicas na retenção de calda sobre a cultura da batata, concluíram que a ponta de jato cônico vazio (JA-4) promoveu maior depósito de calda no terço inferior das plantas, em relação à ponta de jato plano (AVI 110 04), diferenciando-se deste trabalho. Contudo, na parte superior das plantas, as duas pontas comportaram-se de maneira semelhante. BAYER et al. (2011), avaliando diferentes formas de pulverização aérea, concluíram que os atomizadores apresentaram maior penetração de gotas em relação a bicos hidráulicos, uma vez que gotas menores são mais eficientemente captadas pelo alvo.

Como não houve diferença entre as formas de aplicação, isso permite inferir que a aplicação aérea foi tão funcional quanto a aplicação terrestre. A deposição foi melhorada quando se adicionou o adjuvante fosfatidilcoline + ácido propiônico à calda de aplicação.

No momento da segunda aplicação, as plantas de batata encontravam-se no início do processo de senescência das folhas. Assim, as plantas estavam com uma área foliar menos densa, permitindo maior penetração de gotas e menor diferença entre as deposições nas folhas superiores e inferiores (Tabela 7). Não houve diferença significativa para nenhum dos fatores avaliados. Nota-se que a diferença entre as deposições teve menor variação (entre 26,86\% e 46,44\%) do que a ocorrida na primeira aplicação (entre $30,84 \%$ e $60,74 \%$ ), na qual havia maior área foliar e, portanto, maior impedimento à penetração das gotas.

TABELA 7. Diferença entre a deposição de calda nas folhas superiores e inferiores da batata, após a segunda aplicação aérea e terrestre, com e sem adjuvante adicionado à calda. Difference between the spray liquid deposition into lower and upper leaves of potato plants, after the second aerial and ground application, with and without adjuvant addition in the spray liquid.

\begin{tabular}{|c|c|c|c|}
\hline \multirow{3}{*}{$\begin{array}{c}\text { Formas e volumes de aplicação (L ha } \\
{ }_{1}{ }^{-}\end{array}$} & \multicolumn{3}{|c|}{ Diferença $(\%)$} \\
\hline & \multicolumn{2}{|c|}{ Adjuvante } & \multirow{2}{*}{ Média } \\
\hline & Sem & Com & \\
\hline Aéreo - 15 & 20,89 & 32,82 & $26,86 \mathrm{~A}$ \\
\hline Aéreo - 30 & 44,63 & 36,29 & $40,46 \mathrm{~A}$ \\
\hline Terrestre - $200 \mathrm{C}^{\prime}$ & 48,84 & 32,01 & $40,43 \mathrm{~A}$ \\
\hline Terrestre - $400 \mathrm{C}$ & 45,91 & 38,34 & $42,12 \mathrm{~A}$ \\
\hline Terrestre - 200 IA" & 24,74 & 32,10 & $28,42 \mathrm{~A}$ \\
\hline Terrestre - 400 IA & 43,81 & 49,07 & $46,44 \mathrm{~A}$ \\
\hline \multirow[t]{3}{*}{ Média } & $38,14 \mathrm{a}$ & $36,77 \mathrm{a}$ & \\
\hline & & $V=57,98 \%$ & \\
\hline & $\mathrm{F}_{\mathrm{F}}=1,066^{\mathrm{ns}}$ & $\mathrm{F}_{\mathrm{A}}=0,048^{\mathrm{ns}}$ & $\mathrm{F}_{\mathrm{FxA}}=0,528^{\mathrm{ns}}$ \\
\hline
\end{tabular}

Os menores valores de deriva foram encontrados nos dois tratamentos terrestres com pontas de jato plano duplo com indução de ar, demonstrando seu potencial para a redução da deriva (Tabela 8). Esse é um dos motivos pelos quais essas pontas vêm sendo muito utilizadas em pulverizações. Elas resultam em menor interferência das condições climáticas, sem alteração significativa na taxa de aplicação, mas com formação de gotas de diâmetros maiores (BAUER et al., 2006). Contudo, o emprego de gotas grossas depende também das exigências do produto a ser aplicado quanto ao nível de cobertura do alvo (BOLLER et al., 2007). 
TABELA 8. Deriva e perdas para o solo após a segunda aplicação aérea e terrestre, com e sem adjuvante adicionado à calda. Drift and spray liquid run-off after the second aerial and ground application, with and without adjuvant addition in the spray liquid.

\begin{tabular}{|c|c|c|c|c|c|c|}
\hline \multirow{3}{*}{$\begin{array}{l}\text { Formas e volumes } \\
\text { de aplicação (L ha } \\
1 \text { ) }\end{array}$} & \multicolumn{3}{|c|}{ Deriva $\left(\mu \mathrm{g} \mathrm{cm}^{-2}\right)$} & \multicolumn{3}{|c|}{ Perdas para o solo $\left(\mu \mathrm{g} \mathrm{cm}^{-2}\right)$} \\
\hline & \multicolumn{2}{|c|}{ Adjuvante } & \multirow{2}{*}{ Média } & \multicolumn{2}{|c|}{ Adjuvante } & \multirow{2}{*}{ Média } \\
\hline & Sem & Com & & Sem & Com & \\
\hline Aérea - 15 & - & - & - & 0,198 & 0,238 & $0,218 \mathrm{~A}$ \\
\hline Aérea - 30 & - & - & - & 0,290 & 0,142 & $0,216 \mathrm{~A}$ \\
\hline Terrestre - $200 \mathrm{C}^{\prime}$ & 0,100 & 0,073 & 0,086 BC & 0,356 & 0,330 & $0,343 \mathrm{~A}$ \\
\hline Terrestre - $400 \mathrm{C}$ & 0,128 & 0,121 & $0,125 \mathrm{C}$ & 0,232 & 0,365 & $0,299 \mathrm{~A}$ \\
\hline Terrestre - 200 IA" & 0,036 & 0,019 & $0,028 \mathrm{~A}$ & 0,330 & 0,192 & $0,261 \mathrm{~A}$ \\
\hline Terrestre - 400 IA & 0,042 & 0,037 & $0,040 \mathrm{AB}$ & 0,359 & 0,382 & $0,370 \mathrm{~A}$ \\
\hline \multirow[t]{3}{*}{ Média } & $0,077 \mathrm{a}$ & $0,062 \mathrm{a}$ & & $0,294 \mathrm{a}$ & $0,275 \mathrm{a}$ & \\
\hline & \multicolumn{3}{|c|}{$\mathrm{CV}=59,21 \%$} & \multicolumn{3}{|c|}{$\mathrm{CV}=53,58 \%$} \\
\hline & $\mathrm{F}_{\mathrm{F}}=9,340 *$ & $F_{A}=0,963^{n}$ & $\mathrm{~F}_{\mathrm{FxA}}=0,120^{\mathrm{ns}}$ & $\mathrm{F}_{\mathrm{F}}=1,420^{\mathrm{ns}}$ & $F_{A}=0,192^{n}$ & $\mathrm{FxA}_{\mathrm{Fx}}=1,012^{\mathrm{ns}}$ \\
\hline
\end{tabular}

'C: ponta de jato cônico vazio; "IA: ponta de jato plano duplo com indução de ar; CV: coeficiente de variação; $F_{F}$ : valor do $F$ calculado para o fator formas e volumes de aplicação; $\mathrm{F}_{\mathrm{A}}$ : valor do $\mathrm{F}$ calculado para o fator adjuvante; $\mathrm{F}_{\mathrm{FxA}}$ : valor do $\mathrm{F}$ calculado para a interação entre os fatores formas e volumes de aplicação e adjuvante. Médias seguidas por letras distintas maiúsculas, nas colunas, e minúsculas, nas linhas, diferem entre si, pelo teste de Tukey, a 0,05 de probabilidade. ** significativo a 0,01 ; $*$ significativo a 0,05 ;

${ }^{n s}$ não significativo. A deriva para os tratamentos aéreos não foi calculada conforme explicado no item material e métodos.

Não houve diferença significativa com relação à deriva quando se adicionou o adjuvante à calda de aplicação. Avaliando-se as perdas de calda para o solo, não houve diferença entre os tratamentos (Tabela 8).

Os menores tamanhos de gotas foram obtidos com os atomizadores rotativos nos volumes de 15 e $30 \mathrm{~L} \mathrm{ha}^{-1}$ de calda (Tabela 9). Em contrapartida, os maiores valores de tamanho de gotas foram produzidos pelas pontas de jato plano duplo com indução de ar (AD-IA/D), nos volumes de 200 e $400 \mathrm{~L} \mathrm{ha}^{-1}$ de calda. De forma semelhante, NUYTTENS et al. (2007, 2009), avaliando o tamanho de gotas de diferentes pontas de pulverização hidráulica, de mesma vazão nominal, observaram que as pontas de jato plano, com indução de ar, produziram os maiores tamanhos de gotas, obtendo-se assim menor propensão das mesmas à deriva.

Assim como na primeira aplicação, o adjuvante não interferiu no $\mathrm{Dv}_{0,5}$ (Tabela 9). Adjuvantes com propriedades surfactantes, como o fosfatidilcoline + ácido propiônico, podem além de reduzir a tensão superficial, diminuir o tamanho das gotas (BARGEL et al., 2006; DI OLIVEIRA, 2008; THEBALDI et al., 2009). Isso pode explicar a não alteração do $\mathrm{Dv}_{0,5}$ das gotas com a adição do adjuvante à calda de pulverização.

A aplicação aérea com $15 \mathrm{~L} \mathrm{ha}^{-1}$ proporcionou a maior percentagem de gotas aspergidas menores que $100 \mu \mathrm{m}$, diferindo dos outros tratamentos (Tabela 9). Com esse tipo de espectro de gotas, existe risco elevado de deriva. Vale ressaltar que, de acordo com a necessidade, pode-se aumentar o tamanho das gotas geradas pelo atomizador rotativo, simplesmente alterando a angulação das pás. Também existem, no mercado, atomizadores rotativos com dispositivos específicos para aumento de tamanho de gota.

A utilização do adjuvante também não interferiu na percentagem de gotas menores que 100 $\mu \mathrm{m}$. A adição de adjuvantes pode alterar o desempenho das aplicações, contudo é muito importante conhecer o funcionamento desses produtos e as implicações de seu uso antes de adquirí-los e utilizá-los (LAN et al., 2007). 
TABELA 9. Diâmetro da mediana volumétrica e percentagem do volume pulverizado composto por gotas com diâmetro inferior a $100 \mu \mathrm{m}$, após a primeira aplicação aérea e terrestre, com e sem adição de adjuvante à calda. Volume median diameter and percentage of spray volume in droplets smaller than $100 \mu \mathrm{m}$ diameter after the second aerial and ground application, with and without adjuvant addition in the spray liquid.

\begin{tabular}{|c|c|c|c|c|c|c|}
\hline \multirow{3}{*}{$\begin{array}{l}\text { Formas e volumes } \\
\text { de aplicação }\left(\mathrm{L} \mathrm{ha}^{-1}\right)\end{array}$} & \multicolumn{3}{|c|}{$\mathrm{Dv}_{0,5}(\mu \mathrm{m})$} & \multicolumn{3}{|c|}{ Gotas $<100 \mu \mathrm{m}(\%)$} \\
\hline & \multicolumn{2}{|c|}{ Adjuvante } & \multirow{2}{*}{ Média } & \multicolumn{2}{|c|}{ Adjuvante } & \multirow{2}{*}{ Média } \\
\hline & Sem & Com & & Sem & Com & \\
\hline Aérea - 15 & 105 & 93 & $99 \mathrm{~A}$ & 43,77 & 62,39 & $53,08 \mathrm{C}$ \\
\hline Aérea - 30 & 123 & 117 & $120 \mathrm{AB}$ & 28,70 & 35,88 & $32,29 \mathrm{~B}$ \\
\hline Terrestre - $200 \mathrm{C}^{\prime}$ & 158 & 175 & $166 \mathrm{BC}$ & 12,37 & 10,50 & $11,44 \mathrm{~A}$ \\
\hline Terrestre - $400 \mathrm{C}$ & 175 & 203 & $189 \mathrm{C}$ & 10,94 & 7,96 & $9,45 \mathrm{~A}$ \\
\hline Terrestre - 200 IA" & 429 & 411 & $420 \mathrm{D}$ & 1,55 & 1,68 & $1,61 \mathrm{~A}$ \\
\hline Terrestre - 400 IA & 424 & 416 & $420 \mathrm{D}$ & 2,23 & 3,28 & $2,76 \mathrm{~A}$ \\
\hline \multirow[t]{3}{*}{ Média } & $236 \mathrm{a}$ & $236 \mathrm{a}$ & & $16,59 \mathrm{a}$ & $20,28 \mathrm{a}$ & \\
\hline & \multicolumn{3}{|c|}{$\mathrm{CV}=14,20 \%$} & \multicolumn{3}{|c|}{$\mathrm{CV}=42,34 \%$} \\
\hline & \multicolumn{3}{|c|}{$\begin{array}{r}\mathrm{F}_{\mathrm{F}}=152,91 * * \quad \mathrm{~F}_{\mathrm{A}}= \\
\mathrm{F}_{\mathrm{FxA}}=0,59^{\mathrm{ns}}\end{array}$} & \multicolumn{3}{|c|}{$\mathrm{F}_{\mathrm{FxA}}=2,17^{\mathrm{ns}}$} \\
\hline
\end{tabular}

'C: ponta de jato cônico vazio; "IA: ponta de jato plano duplo com indução de ar; $\mathrm{CV}$ : coeficiente de variação; $\mathrm{F}_{\mathrm{F}}$ : valor do $\mathrm{F}$ calculado para o fator formas e volumes de aplicação; $\mathrm{F}_{\mathrm{A}}$ : valor do $\mathrm{F}$ calculado para o fator adjuvante; $\mathrm{F}_{\mathrm{FxA}}$ : valor do $\mathrm{F}$ calculado para a interação entre os fatores formas e volumes de aplicação e adjuvante. Médias seguidas por letras distintas maiúsculas, nas colunas, e minúsculas, nas linhas, diferem entre si, pelo teste de Tukey, a 0,05 de probabilidade. ** significativo a 0,$01 ; *$ significativo a 0,05 ;

${ }^{n s}$ não significativo.

\section{CONCLUSÕES}

A adição do adjuvante fosfatidilcoline + ácido propiônico, de forma geral, melhorou a deposição de calda nas partes superior e inferior das plantas da batata, mas não alterou o diâmetro da mediana volumétrica das gotas e a percentagem do volume composto por gotas menores que 100 $\mu \mathrm{m}$.

A utilização de volumes de calda de 15 ou $30 \mathrm{~L} \mathrm{ha}^{-1}$, em aplicações aéreas, ou $200 \mathrm{~L} \mathrm{ha}^{-1}$ nas aplicações terrestres, proporcionou deposição de calda no dossel semelhante ao volume tradicional de $400 \mathrm{~L} \mathrm{ha}^{-1}$.

A aplicação aérea mostrou-se semelhante às aplicações terrestres estudadas no que se refere à deposição de calda na cultura da batata, mas proporcionou maior percentagem de gotas sujeitas à deriva (menores que $100 \mu \mathrm{m}$ ) nos dois volumes de aplicação estudados.

As pontas de leque duplo com indução de ar propiciaram deposição semelhante às de jato cônico vazio no terço inferior e menor risco de deriva.

\section{AGRADECIMENTOS}

Ao CNPq, à Fapemig e à Capes, pelo suporte financeiro, e ao grupo Rocheto, pela cessão da área experimental, que permitiram o desenvolvimento deste trabalho de pesquisa.

\section{REFERÊNCIAS}

BARGEL, H.; KOCH, K.; CERMAN, Z.; NEINHUIS, C. Structure-function relationships of the plant cuticle and cuticular waxes - a smart material? Functional Plant Biology, Evans Review, Victoria, v. 33, n. 10, p. 893-910, oct. 2006.

BAUER, F. C.; RAETANO, C. G.; PEREIRA, F. A. R. Padrões de distribuição volumétrica de pontas de pulverização de jato plano 11002, com e sem indução de ar, sob diferentes espaçamentos e alturas. Engenharia Agrícola, Jaboticabal, v. 26, n. 2, p. 546-551, maio/ago. 2006. 
BAYER, T.; COSTA, I. F. D; LENZ, G.; ZEMOLIN, C., MARQUES, L.M.; STEFANELO, M. Equipamentos de pulverização aérea e taxas de aplicação de fungicida na cultura do arroz irrigado. Revista Brasileira de Engenharia Agrícola e Ambiental, Campina Grande, v.15, n.2, p.192-198, 2011.

BOLLER, W.; FORCELINI, L. A.; HOFFMANN, L. L. Tecnologia de aplicação de fungicidas Parte I. Revisão Anual de Patologia de Plantas, Passo Fundo, v. 15, p. 243-276, 2007.

CUNHA, J. P. A. R.; FARNESE, A. C.; OLIVET, J. J.; VILLALBA, J. Deposição de calda pulverizada na cultura da soja promovida pela aplicação aérea e terrestre. Engenharia Agrícola, Jaboticabal, v. 31, n. 2, p. 343-351, mar./abr. 2011.

CUNHA, J. P. A. R da; SILVA, R. A. M. Uniformidade de distribuição volumétrica de pontas de pulverização em função da pressão de trabalho e altura da barra. Bioscience Journal, Uberlândia, v. 26, n. 1, p. 52-58, jan./fev. 2010.

CUNHA, J. P. A. R.; TEIXEIRA, M. M.; VIEIRA, R. F.; FERNANDES, H. C. Deposição e deriva de calda fungicida aplicada em feijoeiro, em função de bico de pulverização e de volume de calda. Engenharia Agrícola e Ambiental, Campina Grande, v. 9, n. 1, p. 133-138, 2005.

CUNHA, J. P. A. R. da; TEIXEIRA, M. M.; COURY, J. R.; FERREIRA, L. R. Avaliação de estratégias para redução da deriva de agrotóxicos em pulverizações hidráulicas. Planta Daninha, Viçosa-MG, v. 21, n. 2, p.325-332, maio/ago. 2003.

DI OLIVEIRA, J. R. G. Cobertura da cultura da soja e deposição de inseticida aplicado com e sem adjuvante e diferentes equipamentos e volumes de calda.2008. $76 \mathrm{f}$. Dissertação de (Mestrado) Faculdade de Ciências Agrárias e Veterinárias, Universidade Estadual Paulista, Jaboticabal, 2008.

FARINHA, J. V.; MARTINS, D.; COSTA, N. V.; DOMINGOS, V. D. Deposição da calda de pulverização em cultivares de soja no estádio R1. Ciência Rural, Santa Maria, v. 39, n. 6, p. 1.7381.744, set. 2009.

FERREIRA, M. C.; FERNANDES, A. P.; ROMÁN, R. A. A. Potencial de deriva das gotas grossas produzidas por pontas de pulverização de energia hidráulica, com caldas de diferentes adjuvantes. In: CONGRESSO BRASILEIRO DE ENGENHARIA AGRÍCOLA, 38., 2009, Juazeiro. Anais... Juazeiro: Associação Brasileira de Engenharia Agrícola, 2009. 1 CD-ROM.

FILGUEIRA, F. A. R. Novo manual de olericultura: Agrotecnologia moderna na produção e comercialização de hortaliças. 3. ed. Viçosa: UFV, 2008. 421 p.

GULER, H.; ZHU, H. E.; KERKSEN, R. C.; YU, Y.; KRAUSE, C. R. Spray characteristics and drift reduction potential with air induction and conventional flat-flan nozzles. Transaction of the $A S A E$, Saint Joseph, v. 50, n. 3, p. 745-754, apr. 2007.

LAN, Y.; HOFFMANN, W. C.; FRITZ, B. K.; MARTINS, D. E.; LOPEZ, L. E. Drift reduction with drift control adjuvants. St. Joseph: ASABE -Annual International Meeting, 2007. 14 p. (Paper, 07-1060).

MENDONÇA, C. G.; RAETANO, C. G.; MENDONÇA, C. G. Tensão superficial estática de soluções aquosas com óleos minerais e vegetais utilizados na agricultura. Engenharia Agrícola, Jaboticabal, v. 27, p. 16-23, 2007. Número especial

MILLER, P. C. H.; BUTLER-ELLIS, M. C. Effects of formulation on spray nozzle performance for applications from ground-based boom sprayers. Crop Protection, Silsoe, v. 19, n. 8, p. 609-615, 2000.

NUYTTENS, D.; DE SCHAMPHELEIRE, M.; VERBOVEN, P.; BRUSSELMAN, E.;

DEKEYSER, D. Droplet size and velocity characteristics of agricultural sprays. Transactions of the $A S A B E$, St. Joseph, v. 52, n. 5, p. 1.471-1.480, sept./oct. 2009.

NUYTTENS, D.; BAETENS, K.; DE SCHAMPHELEIRE, M.; SONCK, B. Effect of nozzle type, 
size and pressure on spray droplet characteristics. Biosystems Engineering, Silsoe, v. 97, n. 3, p. 333-345, july 2007.

OZEKI, Y. Manual de aplicação aérea. São Paulo: Ciba Agro, 2006. 101 p.

PALLADINI, L. A.; RAETANO, C. G.; VELINI, E. D. Choice of tracers for the evaluation of spray deposits. Scientia Agricola, Piracicaba, v. 62, n. 5, p. 440-445, sept./oct. 2005.

PINTO, J. R.; LOECK, A. E.; SOUZA, R.T. de; LOUZADA, R. S. Estabilidade à exposição solar dos traçantes azul brilhante e amarelo tartrasina utilizados em estudos de deposição de pulverização. Revista Brasileira de Agrociência, Pelotas, v. 13, n. 1, p. 105-107, 2007.

RYCKAERT, B.; SPANOGHE, P.; HAESAERT, G.; HEREMANS, B.; ISEBAERT, S.; STEURBAUT, W. Quantitative determination of the influence of adjuvants on foliar fungicide residues. Crop Protection, London, v. 26, n. 10, p. 1.589-1.594, oct. 2007.

SCUDELER, F.; FUGIKAWA, L. H.; RAETANO, C. G. Influência do tipo de ponta de pulverização e do volume de aplicação na deposição do traçador cobre na cultura da batata (Solanum tuberosum L.). In: SIMPÓSIO INTERNACIONAL DE TECNOLOGIA DE APLICAÇÃO DE AGROTÓXICOS, 3., 2004, Botucatu. Resumos... Botucatu: Instituto Agronômico de Campinas, 2004. p. 179-181. 1 CD-ROM

THEBALDI, M. S.; REIS, E. F. DOS; GRATÃO, P. T. S.; SANTANA, M S. Efeito da adição de adjuvante na redução de deriva em pontas de pulverização tipo cone vazio. Revista Ciências Técnicas Agropecuárias, Havana , v. 18, n. 2, 2009.

VAN ZYL, S. A.; BRINK, J. C.; CALITZ. F. J.; COERTZE, S.; FOURIE, P. H. The use of adjuvants to improve spray deposition and Botrytis cinerea control on chardonnay grapevine leaves. Crop Protection, London, v. 29, n. 1, p. 58-67, jan. 2010. 